\title{
Temperature Dependence of Torsional Frequencies in Some Dichloro-Anilines Using Nuclear Quadrupole Resonance Data
}

\author{
H.D. Basavegowda And K. Rukmani* \\ Department of Physics, Bangalore University, Bangalore 560056, India
}

(Received August 25, 2006)

\begin{abstract}
The temperature variation of the nuclear quadrupole resonance frequencies in 3,4-dichloro-aniline and 2,6-dichloro-aniline were fitted to three different models available in literature, namely the Kushida model, the Brown model, and a fourth order polynomial. Brown's model with $T_{0}=205 \mathrm{~K}$ gave the best fit in the case of both compounds indicating that the temperature dependence of the torsional frequencies cannot be neglected in these compounds. The internal torsional frequencies were evaluated from the nuclear quadrupole resonance data by using a two-mode approximation in a temperature range of $77-300 \mathrm{~K}$ for the above two compounds. The torsional frequencies lie in a range of $30-60 \mathrm{~cm}^{-1}$ in both compounds and agree closely with the Raman lines obtained in 2,6-dichloro-aniline. The lower frequency mode around $37 \mathrm{~cm}^{-1}$ may be associated with the motion around an axis perpendicular to the benzene ring which is present in both compounds.
\end{abstract}

PACS numbers: $76.60 . \mathrm{Gv}$

\section{Introduction}

The nuclear quadrupole resonance (NQR) frequencies in molecular solids are known to be affected by the internal bond bending motions, giving rise to their temperature variation. Hence a study of the temperature variation of the NQR frequencies can provide information on the internal torsional frequencies and their temperature variation. The dichloro-anilines are small benzene ring molecules with an amino group and two chlorines substituted on the ring. The chlorine on the ring is a suitable probe for NQR and the effect of substitution on the torsional frequencies may be evaluated by a study of such compounds. Two such molecular solids namely 3,4-dichloro-aniline and 2,6-dichloro-aniline were chosen and the ${ }^{35} \mathrm{Cl} \mathrm{NQR}$ data was used to obtain the internal torsional frequencies in these compounds.

*corresponding author; e-mail: rukmani9909@yahoo.co.in 


\section{Temperature dependence of NQR frequencies}

The variation of NQR frequency with temperature can be fitted to three models available in literature [1-3]. The functional form obtained by Kushida et al. [1] from Bayer's theory [2] under high temperature approximation is

$$
\nu(T)=A_{-1} / T+A_{0}+A_{1} T .
$$

Brown [3] assumed a linear temperature dependence for the torsional frequencies and obtained an expression of the form

$$
\nu(T)=A_{0}+A_{1}\left(T-T_{0}\right)+A_{2}\left(T-T_{0}\right)^{2},
$$

where $T_{0}$ is an arbitrary reference temperature in the high temperature region.

The temperature variation of the NQR frequency can also be attempted to fit a general fourth order polynomial of the form given below

$$
\nu(T)=A_{0}+A_{1} T+A_{2} T^{2}+A_{3} T^{3}+A_{4} T^{4} .
$$

The NQR spectra of 3,4-dichloro-aniline as observed by Nakayama et al. [4] show two lines throughout a temperature range of 77-300 K. The higher frequency line (line II) may be assigned to the 3-chlorine while the lower frequency line (line I)

TABLE I

Least squares fit parameters and $\langle g\rangle$ corresponding to line I of 3,4-dichloro-aniline.

\begin{tabular}{c|c|c|c}
\hline \hline Parameters & 4 th order polynomial & Brown $T_{0}=205 \mathrm{~K}$ & Kushida \\
\hline$A_{-1}[\mathrm{MHz} \mathrm{K}]$ & - & - & $0.3529 \times 10^{-2}$ \\
$A_{0}[\mathrm{MHz}]$ & 35.79 & 35.39 & 35.39 \\
$A_{1}\left[\mathrm{MHz} \mathrm{K}^{-1}\right]$ & $-0.1321 \times 10^{-2}$ & $-0.2686 \times 10^{-2}$ & $-0.1054 \times 10^{-2}$ \\
$A_{2}\left[\mathrm{MHz} \mathrm{K}^{-2}\right]$ & $-0.2604 \times 10^{-5}$ & $0.3028 \times 10^{-5}$ & - \\
$A_{3}\left[\mathrm{MHz} \mathrm{K}^{-3}\right]$ & $-0.2831 \times 10^{-8}$ & - & - \\
$A_{4}\left[\mathrm{MHz} \mathrm{K}^{-4}\right]$ & $0.1666 \times 10^{-11}$ & - & - \\
$\Delta\left[\mathrm{MHz}^{2}\right]$ & $0.4585 \times 10^{-4}$ & $0.3659 \times 10^{-4}$ & $0.1871 \times 10^{-2}$ \\
$\langle g\rangle\left[\mathrm{K}^{-1}\right]$ & $0.804086 \times 10^{-3}$ & 0.000592 & -
\end{tabular}

TABLE II

Least squares fit parameters and $\langle g\rangle$ corresponding to line II of 3,4-dichloro-aniline.

\begin{tabular}{c|c|c|c}
\hline \hline Parameters & 4 th order polynomial & Brown $T_{0}=205 \mathrm{~K}$ & Kushida \\
\hline$A_{-1}[\mathrm{MHz} \mathrm{K}]$ & - & - & $0.4955 \times 10^{-2}$ \\
$A_{0}[\mathrm{MHz}]$ & 35.05 & 35.45 & 35.45 \\
$A_{1}\left[\mathrm{MHz} \mathrm{K}^{-1}\right]$ & $-0.2035 \times 10^{-2}$ & $-0.3846 \times 10^{-2}$ & $-0.1515 \times 10^{-2}$ \\
$A_{2}\left[\mathrm{MHz} \mathrm{K}^{-2}\right]$ & $-0.3918 \times 10^{-5}$ & $-0.3402 \times 10^{-5}$ & - \\
$A_{3}\left[\mathrm{MHz} \mathrm{K}^{-3}\right]$ & $-0.3661 \times 10^{-8}$ & - & - \\
$A_{4}\left[\mathrm{MHz} \mathrm{K}^{-4}\right]$ & $0.6882 \times 10^{-11}$ & - & - \\
$\Delta\left[\mathrm{MHz}^{2}\right]$ & $0.9012 \times 10^{-4}$ & $0.7248 \times 10^{-4}$ & $0.3654 \times 10^{-2}$ \\
$\langle g\rangle\left[\mathrm{K}^{-1}\right]$ & $0.647672 \times 10^{-3}$ & 0.0004602 & -
\end{tabular}


may be assigned to the 4-chlorine using the additive model of the substitution effect [5]. The results of the iterative least squares fitting of the NQR data for the two lines are given in Tables I and II. $\Delta$ is the root mean square deviation and $\langle g\rangle$ is the average temperature coefficient. It is seen from the $\Delta$ values that Brown's parabolic curve equation gives the best fit in both cases. Since Brown's equation was derived using a linear temperature variation of the torsional frequencies, the temperature variation of the torsional frequencies cannot be neglected in this compound.

The NQR data of 2,6-dichloro-aniline [6] also show two lines in a temperature range of $80-240 \mathrm{~K}$. Both the chlorines 2 and 6 are symmetric with respect to the $\mathrm{NH}_{2}$ and are expected to have the same frequency if the additive model is used. Since two NQR lines are observed, it can be concluded that steric effects render the two chlorines inequivalent. Therefore the higher frequency line (line II) may be assigned to 2-chlorine and the lower frequency line (line I) to 6-chlorine. The least squares fit results of this data are given in Tables III and IV. Brown's equation

TABLE III

Least squares fit parameters and $\langle g\rangle$ corresponding to line I of 2,6-dichloro-aniline.

\begin{tabular}{c|c|c|c}
\hline \hline Parameters & 4 th order polynomial & Brown $T_{0}=205 \mathrm{~K}$ & Kushida \\
\hline$A_{-1}[\mathrm{MHz} \mathrm{K}]$ & - & - & $0.1751 \times 10^{-2}$ \\
$A_{0}[\mathrm{MHz}]$ & 34.50 & 39.94 & 33.96 \\
$A_{1}\left[\mathrm{MHz} \mathrm{K}^{-1}\right]$ & $-0.1371 \times 10^{-2}$ & $-0.4439 \times 10^{-2}$ & $-0.1349 \times 10^{-2}$ \\
$A_{2}\left[\mathrm{MHz} \mathrm{K}^{-2}\right]$ & $-0.4215 \times 10^{-5}$ & $-0.7582 \times 10^{-5}$ & - \\
$A_{3}\left[\mathrm{MHz} \mathrm{K}^{-3}\right]$ & $-0.9231 \times 10^{-8}$ & - & - \\
$A_{4}\left[\mathrm{MHz} \mathrm{K}^{-4}\right]$ & $-0.1277 \times 10^{-10}$ & - & - \\
$\Delta\left[\mathrm{MHz}^{2}\right]$ & $0.4072 \times 10^{-4}$ & $0.1311 \times 10^{-4}$ & $0.1712 \times 10^{-2}$ \\
$\langle g\rangle\left[\mathrm{K}^{-1}\right]$ & $0.157920 \times 10^{-2}$ & 0.0009153 & -
\end{tabular}

TABLE IV

Least squares fit parameters and $\langle g\rangle$ corresponding to line II of 2,6-dichloro-aniline.

\begin{tabular}{c|c|c|c}
\hline \hline Parameters & 4 th order polynomial & Brown $T_{0}=205 \mathrm{~K}$ & Kushida \\
\hline$A_{-1}[\mathrm{MHz} \mathrm{K}]$ & - & - & $0.1499 \times 10^{-2}$ \\
$A_{0}[\mathrm{MHz}]$ & 35.08 & 34.60 & 34.61 \\
$A_{1}\left[\mathrm{MHz} \mathrm{K}^{-1}\right]$ & $-0.1027 \times 10^{-2}$ & $-0.4092 \times 10^{-2}$ & $-0.1197 \times 10^{-2}$ \\
$A_{2}\left[\mathrm{MHz} \mathrm{K}^{-2}\right]$ & $-0.3454 \times 10^{-5}$ & $-0.8931 \times 10^{-5}$ & - \\
$A_{3}\left[\mathrm{MHz} \mathrm{K}^{-3}\right]$ & $-0.9256 \times 10^{-8}$ & - & - \\
$A_{4}\left[\mathrm{MHz} \mathrm{K}^{-4}\right]$ & $-0.2081 \times 10^{-10}$ & - & - \\
$\Delta\left[\mathrm{MHz}^{2}\right]$ & $0.1980 \times 10^{-4}$ & $0.1485 \times 10^{-4}$ & $0.1463 \times 10^{-2}$ \\
$\langle g\rangle\left[\mathrm{K}^{-1}\right]$ & $0.190205 \times 10^{-2}$ & 0.0011869 & -
\end{tabular}


gives the best fit for this compound indicating that the temperature dependence of the torsional frequencies is important in this case, too.

\section{Torsional frequency calculations}

Bayer's theory [2] for the temperature dependence of NQR frequency modified by Tatsuzaki and Yokozawa [7] gives

$$
\nu(T)=\nu_{0}\left\{1-\frac{3 h}{8 \pi^{2} c} \sum_{i} \frac{\sin ^{2} \alpha_{i}}{A_{i} f_{i}}\left[\frac{1}{2}+\frac{1}{\exp \left(h f_{i} c / k T\right)-1}\right]\right\},
$$

where $\nu(T)$ is the NQR frequency at temperature $T, \nu_{0}$ is the NQR frequency at $0 \mathrm{~K}, A_{i}$ 's represent the moment of inertia about $x$ and $y$ directions respectively, the $f_{i}$ 's are the corresponding torsional frequencies, $\alpha_{i}$ is the angle between the principal axis of the electric field gradient tensor and the principal axes of the moment of inertia tensor, $h$ is the Planck constant, $c$ - the velocity of light, $k-$ the Boltzmann constant and $T$ - the absolute temperature.

$\nu_{0}$ can be obtained from the least squares fit data, $A_{x}$ and $A_{y}$ from the molecular conformation and the torsional frequencies $f_{x}$ and $f_{y}$ may then be calculated from Eq. (4). The moments of inertia $A_{x}$ and $A_{y}$ were calculated assuming a planar molecule with a regular hexagon for the benzene ring and standard bond lengths of $\mathrm{C}-\mathrm{C}=140 \mathrm{pm}, \mathrm{C}-\mathrm{Cl}=172 \mathrm{pm}, \mathrm{C}-\mathrm{N}=130 \mathrm{pm}$. The contribution of the protons to the moments of inertia are small and were neglected. Table $\mathrm{V}$ lists their values.

TABLE V

Moment of inertia values in 3,4-dichloro-aniline and 2,6-dichloro-aniline.

\begin{tabular}{|c|c|c|}
\hline & $A_{x}\left[\times 10^{-40} \mathrm{~g} \mathrm{~cm}^{2}\right]$ & $A_{y}\left[\times 10^{-40} \mathrm{~g} \mathrm{~cm}^{2}\right]$ \\
\hline \multicolumn{3}{|c|}{ 3,4-dichloro-aniline } \\
\hline line I & 530.96 & 1139.42 \\
\hline line II & 799.20 & 1232.71 \\
\hline \multicolumn{3}{|c|}{ 2,6-dichloro-aniline } \\
\hline line I & 787.56 & 1268.52 \\
\hline line II & 787.56 & 1268.52 \\
\hline
\end{tabular}

A two-mode approximation [8-11] was used and the torsional frequencies $f_{x}$ and $f_{y}$ were calculated at each temperature for lines I and II of each compound. This temperature dependence of the torsional frequencies is shown in Figs. 1 to 4. 

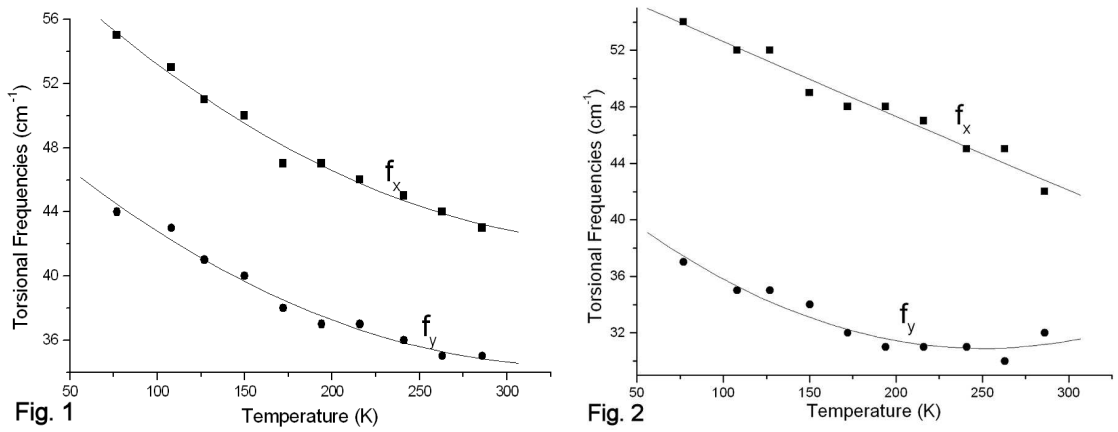

Fig. 1. Temperature variation of the torsional frequencies calculated from NQR line I in 3,4-dichloro-aniline.

Fig. 2. Temperature variation of the torsional frequencies calculated from NQR line II in 3,4-dichloro-aniline.
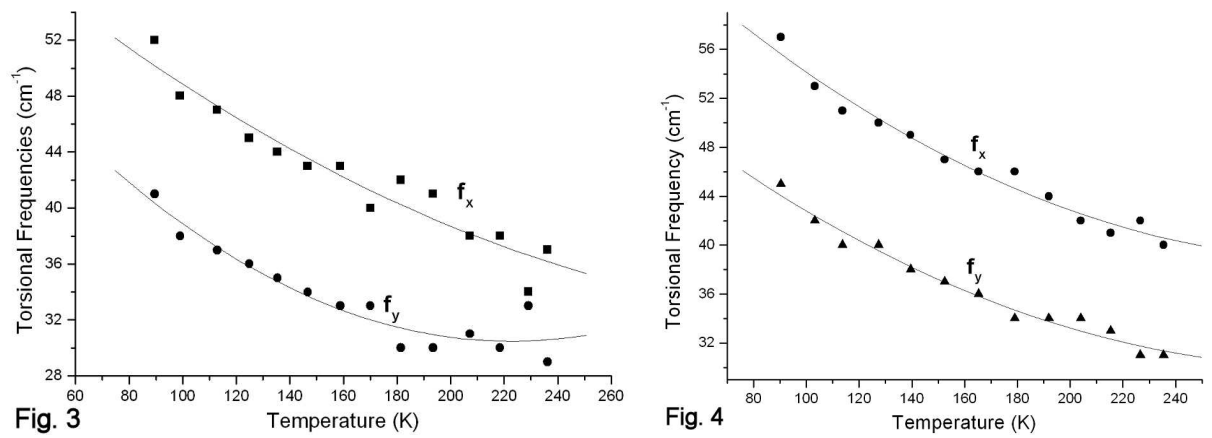

Fig. 3. Temperature variation of torsional frequencies calculated from NQR line I of 2,6-dichloro-aniline.

Fig. 4. Temperature variation of torsional frequencies calculated from NQR line II of 2,6-dichloro-aniline.

\section{Results and conclusions}

The torsional frequencies and their temperature dependence obtained from both NQR lines of 3,4-dichloro-aniline are similar to that obtained from 2,6-dichloro-aniline and lie between $30 \mathrm{~cm}^{-1}$ and $60 \mathrm{~cm}^{-1}$. In both compounds, the lower frequency mode varies between $29 \mathrm{~cm}^{-1}$ and $45 \mathrm{~cm}^{-1}$ and the higher frequency mode between $40 \mathrm{~cm}^{-1}$ and $57 \mathrm{~cm}^{-1}$ corresponding to the two NQR lines. In 2,5-dichloro-aniline, which is similar to the two compounds studied by us, the torsional frequencies obtained using a single mode approximation [12] are $40.45 \mathrm{~cm}^{-1}$ and $42.38 \mathrm{~cm}^{-1}$ for line I and line II, respectively. This is in agreement with our results. Raman studies on 2,6-dichloro-aniline [6] detected four low frequency modes at $95,83,53$, and $32 \mathrm{~cm}^{-1}$ active at temperatures above $220 \mathrm{~K}$. The torsional frequencies calculated by us in the same compound are in close agreement with the last two of the above. The lower frequency mode around 
$37 \mathrm{~cm}^{-1}$ will correspond to motion about the highest moment of inertia axis and may be identified with motion around an axis perpendicular to the ring. This is expected to be present in all the dichloro-anilines.

\section{Acknowledgments}

This work was supported in part by financial assistance from UGC under a project.

\section{References}

[1] T. Kushida, G. Benedek, N. Bloembergen, Phys. Rev. 104, 1364 (1956).

[2] H. Bayer, Z. Phys. 130, 227 (1951).

[3] R.J.C. Brown, J. Chem. Phys. 32, 116 (1960).

[4] H. Nakayama, N. Nakamura, H. Chihara, J. Magn. Reson. 53, 103 (1983).

[5] D. Biedenkapp, A. Weiss, Ber. Bunsenges. Physik. Chem. 70, 788 (1966).

[6] M.E. Ramia, M.J. Zuriaga, C.A. Martin, J. Phys. C, Solid State Phys. 21, 3467 (1988).

[7] I. Tatsuzaki, Y. Yokozawa, J. Phys. Soc. Jpn. 12, 802 (1957).

[8] M.S. Vijaya, J. Ramakrishna, J. Mol. Phys. 19, 131 (1970).

[9] V.S.S. Shastry, J. Ramakrishna, Indian J. Pure Appl. Phys. 15, 658 (1977).

[10] K. Rukmani, Ph.D. Thesis, Indian Institute of Science, 1983.

[11] H.D. Basavegowda, M.Phil. Thesis, Bangalore University, 1994.

[12] J. Ramakrishna, Proc. Phys. Soc. Lond. 86, 595 (1965). 